and non-lasing ones - are affected, thus eliminating mode competition or mode hopping. The mechanism broadly tunes the entire lasing mode until its spectral or spatial overlap with the gain material becomes too weak, in which case the most favoured Fabry-Pérot modes will take over.

Pulling or pushing the plungers by $\sim 25 \mu \mathrm{m}$ achieves red and blue shifts, respectively, which together give a total tuning range of $\sim 137 \mathrm{GHz}$ (3.6\%) of the centre wavelength. Theory estimates that $430 \mathrm{GHz}(11 \%)$ of the centre wavelength is possible. This is achieved without using any other tuning mechanisms such as temperature, current or voltage; these could also be added to the scheme to complement the mechanical tuning as they are fundamentally unrelated to it.

The advantage of this new tuning concept, demonstrated here for terahertz QCLs, is that it exploits the fact that the large wavelength is similar to the length scales of fine mechanics, piezo-electric drives and micro-electromechanical systems. Furthermore, it is continuous and broad enough to cover the laser's entire gain spectrum. When a more sturdy (stick-slipfree) mechanical assembly is found and implemented, opportunities abound to provide more varied and more rapidly changing optical environments for the mechanical tuning of these lasers.
Hence, broader tuning ranges should be possible in a relatively simple manner.

Claire Gmachl is at the Department of Electrical Engineering and MIRTHE at Princeton University, Princeton, New Jersey 08544, USA.

e-mail: cgmachl@princeton.edu

References

1. Qin, Q., Williams, B. S, Kumar, S., Reno, J. L. \& Hu, Q Nature Photon. 3, 732-737 (2009)

2. Köhler, R. et al. Nature 417, 156-159 (2002).

3. Williams, B. S., Kumar, S., Hu, Q. \& Reno, J. L. Opt. Lett. 30, 2909-2911 (2005).

4. Freeman, J. R, Marshall, O. P., Beere, H. E. \& Ritchie, D. A Opt. Express 16, 19830-19835 (2008).

5. Neukermans, A. \& Ramaswami, R. IEEE Commun. Mag. 39, 62-69 (2001)

6. Orlova, E. E. et al. Phys. Rev. Lett. 96, 173904 (2006).

\title{
PHOTOVOLTAICS
}

\section{Solar success for Sharp}

Sharp's activities in photovoltaics have recently provided the company with two pieces of good news. The first is that its research labs in Japan have fabricated a triple-junction solar cell with a recordbreaking power conversion efficiency of $35.8 \%$ - the highest value reported for any cell illuminated with unconcentrated sunlight. The second is that a solarpowered vehicle covered with Sharp's compound solar cells triumphed in the Global Green Challenge solar-car race across Australia at the end of October. The 3,000-km race from Darwin to Adelaide was won by a team from Tokai University in Japan. Their 'Tokai Challenger' vehicle completed the course with an average speed of approximately $100 \mathrm{~km} \mathrm{~h}^{-1}$.

Sharp's record-breaking

$35.8 \%$-efficiency cells consist of three vertically stacked $p-n$ junctions that are each made from a different compound semiconductor (the top, middle and bottom layers are InGaP, GaAs and InGaAs, respectively). Each junction is designed to absorb light at a different wavelength band of the solar spectrum, which maximizes power generation. Such multijunction cells have been a popular design for many years and are often used in space to help power satellites, and in solar farms, which use reflectors to concentrate the intensity of sunlight. Indeed, when used with concentrated sunlight, triple-junction cells - developed independently at Sharp, the Fraunhofer Institute for Solar Energy in Germany and the US firm

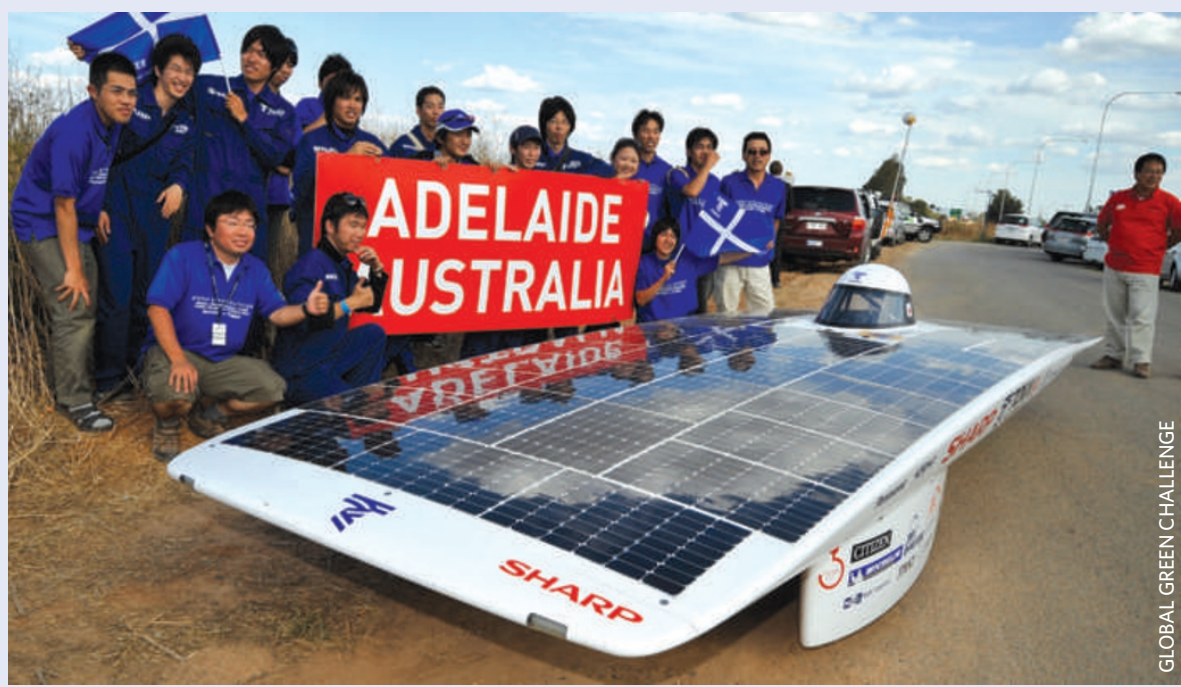

Spectrolab - all achieved efficiencies of over $40 \%$, but this figure is usually much lower for unconcentrated sunlight.

According to Sharp, the key to achieving these latest results for unconcentrated light was to form the bottom junction of the cell from InGaAs rather than germanium - a challenge only recently solved through considerable improvements in semiconductor fabrication technology. The research that enabled this development took place under a solar-cell initiative organized by Japan's New Energy and Industrial Technology Development Organization.

Unfortunately, it was not all good news in October for Sharp. The firm's biannual business results for the six months ending 30 September 2009 showed a year-onyear drop of $17.5 \%$ in net sales to 1,288 billion yen. Worse still, operating income declined $96.9 \%$ from 50.8 billion yen in 2008 to 1.5 billion yen in 2009 for the same biannual period, only narrowly avoiding a net loss. Sales of LCDs (down 33\%) and other electronic items (down 27\%) were particularly hard hit owing to the global recession, but the firm saw sales rise from June onwards and is now benefiting from a cost-reduction programme that it implemented earlier in the year.

\section{OLIVER GRAYDON}

